\title{
Jacques VI, dernier éclat culturel autochtone de la Cour d'Écosse
}

James VI and the Last Native Cultural Flowering of the Scottish Court

Jacques Tranier

\section{(2) OpenEdition}

12 Journals

\section{Édition électronique}

URL : https://journals.openedition.org/etudesecossaises/650

DOI : 10.4000/etudesecossaises.650

ISSN : 1969-6337

Éditeur

UGA Éditions/Université Grenoble Alpes

\section{Édition imprimée}

Date de publication : 15 avril 2012

Pagination : 113-129

ISBN : 978-2-84310-223-3

ISSN : 1240-1439

Référence électronique

Jacques Tranier, « Jacques VI, dernier éclat culturel autochtone de la Cour d'Écosse », Études écossaises [En ligne], 15 | 2012, mis en ligne le 15 octobre 2013, consulté le 11 avril 2023. URL : http:// journals.openedition.org/etudesecossaises/650 ; DOI : https://doi.org/10.4000/etudesecossaises. 650 


\section{Jacques VI, dernier éclat culturel autochtone de la Cour d'Écosse}

\section{Un contexte géo-politique a priori peu propice à la culture savante}

Maints facteurs ont joué contre la culture aristocratique écossaise. Isolé par les intempéries la moitié de l'année, l'Écosse, pauvre en ressources, se trouvait géographiquement éloigné du continent. Dans le contexte de lutte acharnée entre Réforme et contre-Réforme, l'Écosse représentait un enjeu stratégique pour les puissances catholiques. La politique intérieure du royaume fut des plus troublée : succession de régents durant la minorité du monarque ${ }^{1}$, manœuvres ourdies autour de la personne de Mary Queen of Scots, emprisonnée à Londres; rivalités entre puissants seigneurs écossais autour de la personne du jeune Jacques VI, couronné à un an et demi en 1567 ; pressions exercées sur la couronne écossaise pour favoriser l'une ou l'autre des deux religions en guerre. Les luttes intestines entre puissants s'exacerbèrent dès 1579 lorsque le monarque, âgé de treize ans, put enfin choisir ses conseillers et compagnons. À partir de 1590, on vit se succéder des factions dans un pays majoritairement protestant mais dont le roi tenait ses distances à l'égard du presbytérianisme. Le souverain devait en outre ménager sa cousine Elisabeth $\mathrm{I}^{\mathrm{re}}$ dans l'espoir d'hériter du trône anglais. On exécuta la mère de Jacques VI en 1587 et une année plus tard l'invincible armada partait à la conquête de l'Angleterre, suivie en 1594 d'une seconde flotte d'invasion.

Le brillant poète de Cour Alexander Montgomerie, calviniste converti au catholicisme puis introduit à la Cour de Philippe II d'Espagne, finit par perdre la protection de Jacques VI en 1584, victime de ses menées pour le compte de la contre-Réforme : il faut y voir un symptôme des turbulences politiques de l'époque. Les artistes, pour exercer leur vocation,

1. Jacques V (1512-1542) et Jacques VI (1566-1625). 
devaient alors s'attirer la protection d'un grand de ce monde. S'ils s'engageaient trop ouvertement dans des activités politiques ou religieuses, ils encouraient des risques ${ }^{2}$.

Les désordres que connut l'Écosse au XVI ${ }^{\mathrm{e}}$ siècle font songer irrésistiblement à ce qu'écrira Thomas Hobbes dans son Leviathan concernant l'absolue nécessité d'un ordre public stable afin que puissent se développer toutes les entreprises humaines, y compris dans le domaine artistique et intellectuel : il faut bien constater que la vie culturelle à la Cour d'Écosse connut de longues éclipses engendrées par les troubles internes et externes travaillant le pays.

De plus, beaucoup des productions de la haute culture écossaises se sont perdues, faute d'avoir été imprimées, ou de se répandre suffisamment en dehors de la Cour. C'est le cas notamment des « masques », spectacles aristocratiques dont les Cours élisabéthaine et jacobéenne, par contre, nous ont légué maints exemples, dans leur intégralité.

D'ailleurs l'Église réformée prit le contrôle des imprimeurs écossais dès 1563 et la censure qu'elle exerça ne fut guère favorable, on s'en doute, à l'art poétique ou musical, car les Presbytériens honnissaient poésie et danse, arts cultivés, horreur suprême, par les Français catholiques. La « vieille alliance » exposait la Cour d'Écosse à de bien pernicieuses influences...

Malgré tout, le règne de Jacques VI permit à l'Écosse de tenir un certain rang dans la vie culturelle européenne, que caractérisait une circulation constante d'œuvres, de créateurs et d'intellectuels. Toutefois, la seule période ayant laissé des productions identifiables et datables couvre une seule décennie, de 1579 à 1590, date à laquelle Jacques VI s'affranchit de la régence et se maria. Il ne reste pas grand-chose de la période 1590-1603, année où la Cour écossaise déménagea à Londres. L'Écosse perdit alors le principe moteur de sa haute culture et ce vide ne fut jamais remplacé; on vit la fin du mécénat royal en Écosse et la disparition d'un public de Cour approprié. Une certaine pratique de la poésie et de la musique perdura cependant dans les manoirs aristocratiques et lieux de savoir, ce qu'attestent des manuscrits survivants contenant poèmes et pièces instrumentales au XVII ${ }^{\mathrm{e}}$ siècle $^{3}$.

2. Ceci rappelle, toute proportion gardée, les conditions prévalant en Union Soviétique, régime où tout créateur ne pouvait exister sans l'aval du pouvoir et devait par conséquent user de ruse pour faire passer ce qui ne cadrait pas avec la ligne idéologique du moment : les rapports tendus que le compositeur Chostakovitch (1906-1975) entretint avec Staline en sont un exemple notoire.

3. The Rowallen Lute Book (avant 1609[?]) — The Skene Mandore manuscript (1615-1635) — The Straloch manuscript (1627-1629) — The Lady Margaret Wemyss Lute Book (1643-1648) — The Balcarres Manuscript (1700) — The Panmure manuscript (milieu XVII ${ }^{\mathrm{e}}$ ). 


\section{La Cour d'Écosse dans le contexte culturel européen : élégance et divertissement}

En 1584, le poète William Fowler détrôna Montgomerie comme poète officiel, en obtenant la faveur royale. Il ouvrit la Cour à l'influence de la poésie italienne. Dès 1590 celle-ci devint prépondérante. Jusqu’à la disgrâce de Montgomerie, c'est l'influence française qui primait, tant dans le domaine de la poésie que dans celui de la danse : l'épouse de Jacques VI, Anne de Danemark, dont certaines dames de compagnie étaient françaises, se montrait friande de divertissements, et notamment de danse, art particulièrement prisé en France.

À la Renaissance on tient la poésie de Cour en haute estime, car elle relève du prestige culturel national. Mais on l'élabore tout autant pour le plaisir, elle est aussi marque d'esprit et délassement intellectuel. On apprécie les énigmes, musicales ou poétiques, alliant le divertissement du jeu à la maîtrise technique. Les poètes de Cour utilisent l'art de la conversation, les nouvelles possibilités offertes par les langues « vulgaires », en l'occurrence le «Middle Scots ». Ils oeuvrent dans un milieu où le gentilhomme doit savoir charmer, amuser et varier ses propos, ce que préconise d'ailleurs le manuel du parfait courtisan, Il Corteggiano de Balthassare Castiglione $^{4}$ : ce n'est pas un hasard si cet ouvrage se présente sous forme de duels oratoires ou discussions spirituelles dont l'enjeu est de déterminer ce que devraient être les qualités du gentilhomme modèle. Il Corteggiano met en avant une qualité essentielle de l'aristocrate : l'élégance et l'absence d'effort apparent dans tout ce qu'il entreprend — d'où le double aspect de la poésie, alliant activité ludique pour l'esprit et réelle compétence du versificateur. Ainsi, en Espagne, on voit des poètes de Cour s'affronter dans le jeu littéraire des «preguntas y respuestas » : un poète pose une devinette en vers, à laquelle doit répondre un autre poète, sur le même mode. L'Écosse n'est pas en reste, mêlant d'ailleurs traditions culturelles ancestrales et pratiques culturelles continentales contemporaines : Alexander Montgomerie supplante le poète de Cour Hume of Polwarth, au terme d'une joute d'invectives dans la plus pure tradition gaélique pré-chrétienne, le roi Jacques VI prenant le rôle d'arbitre! Le précepteur de Jacques V, Sir Henry Lindsay, notait déjà avec quel bonheur son élève faisait assaut d'esprit contre ceux de son entourage.

4. 1528, Venise, avec une quarantaine d'éditions italiennes au Xvi siècle, des traductions en six langues éditées dans vingt centres urbains européens et une centaine d'éditions en Europe au $\mathrm{XVII}^{\mathrm{e}}$ siècle. L'ouvrage fut traduit en anglais en 1561 par Thomas Hoby, pour ensuite grandement influencer les élites anglaises. On considère $I l$ Corteggiano comme la quintessence de l'esprit de Cour à la Renaissance, élevé au rang de manuel de savoir-vivre destiné à l'entourage des grands de ce monde. 
D'autre part, la Cour d'Écosse suit aussi les pratiques culturelles continentales en mettant à l'honneur la poésie lors de célébrations ou rites publics qui en renforcent la solennité. Ainsi, à l'occasion du nouvel an 1583-1584, on célèbre le roi-poète Jacques VI qui se voit offrir une couronne de lauriers en or, don du poète-lauréat John Stewart of Baldyneis, accompagnée d'un sonnet intitulé In Praise of the Art of Poesie. Ce type de rituel, sur le modèle du couronnement de Pétrarque en Italie ${ }^{5}$, n'était pas unique en Europe : en 1491 l'empereur du Saint Empire germanique Frédéric III couronnait l'humaniste et érudit poète latin Conrad Celtis à son retour d'Italie.

Les ballets de Cour et « masques » extravagants mêlant danse, poésie, mime, costumes, décors et musique, représentaient le microcosme du royaume, d'après Francis Bacon ${ }^{6}$. Ainsi, un masque sur le thème de la paix était-il censé conjurer la guerre civile et son cortège de maux. Ces spectacles, éminemment allégoriques, avaient pour figure centrale la personne du monarque, cœur du pouvoir et garant de la prospérité du royaume : on constate encore l'alliance du divertissement destiné aux sens et du sérieux apporté à l'objet représenté. Ce type de divertissement était aussi l'occasion pour la Cour de faire bombance, et pour certains courtisans, de participer masqués aux côtés de danseurs professionnels ${ }^{7}$. Il s'agissait pour la Cour de se donner elle-même en représentation, si possible en présence d'ambassadeurs et autres invités étrangers de marque, à des fins de prestige et de propagande. Le masque, occasion de flatter le souverain, traitait un thème lié à l'actualité ou au calendrier sous l'aspect de la mythologie antique. Malheureusement, et ceci s'avère très frustrant, absolument rien ne subsiste des masques donnés à la Cour d'Écosse, contrairement à ceux qui distrayaient celle d'Angleterre ${ }^{8}$.

5. Le 8 avril 1341, jour de Pâques, au Capitole de Rome, lors d'une cérémonie fastueuse, Pétrarque reçoit du sénateur Orso dell'Anguillara, la «Couronne de laurier d'Apollon » le consacrant Roi des poètes. Cette consécration assit la réputation de Pétrarque dans toute l'Europe.

6. «Of Masques and Triumphs », dans Essayes or Counsels, Civill and Morall (1625).

7. De la même manière, en France, Louis XIV, excellent danseur, se produira dans des ballets de cour à Versailles.

8. Il nous reste au moins les textes de dizaines de masques à l'époque de Jacques $\mathrm{I}^{\text {er }}$ et Charles $\mathrm{I}^{\mathrm{er}}$, œuvres de Ben Johnson, Thomas Campion, George Chapman, Thomas Carew, Samuel Daniel, Aurelian Townsend, William Davenant. Ces documents, ainsi que les fragments de musique, les planches illustrant les costumes et décors, nous donnent rétrospectivement, de manière indirecte certes, une idée de leur équivalent écossais avant 1603. Les sommes dépensées en pareille occasion pouvaient atteindre des montants faramineux : le masque donné par Lord Hay en l'honneur de l'ambassadeur français en 1616 coûta en monnaie d'alors $£ 2$ 200, banquet compris; Bacon lui-même consacra $£ 2000$ au Masque of Flowers (1613). On sait que George Buchanan composa une chanson, Offering of the Rustic Gods, destinée au masque donné le soir du baptême de Jacques VI le 17 décembre 1566 au château de Stirling. C'est le Français Bastian Pagez, serviteur et musicien de Mary reine d'Écosse, qui organisa une partie des festivités. 


\section{Jacques VI et l'influence civilisatrice des arts}

La violence est chose courante à l'époque, et l'Écosse connaît sa part d'infortunes : on se souvient que le roi Jacques IV périt avec la fine fleur de l'aristocratie écossaise à la bataille de Flodden contre les Anglais ${ }^{9}$. Jacques $\mathrm{V}$ et son successeur Jacques VI connurent un sort identique : ils accédèrent au trône encore bébés; ils virent le pouvoir tomber aux mains de régents et partis divers; on les séquestra un temps avant qu'ils puissent enfin exercer le pouvoir ${ }^{10}$.

Jacques VI, esprit éclectique, fait partie des princes de la Renaissance qui reçurent une solide culture. Lui-même poète, il connaissait dans le texte les versificateurs français grâce à la bibliothèque de sa mère Mary Queen of Scots, dont la jeunesse avait eu pour cadre la Cour de France. D'autre part, son précepteur Buchanan lui avait excellemment enseigné l'italien, outre le français. Buchanan avait effectué de nombreux séjour en France pour y occuper diverses fonctions ${ }^{11}$, fréquenter érudits et poètes ${ }^{12}$. Jacques $\mathrm{V}$, bon poète et luthiste, avait bénéficié d'une éducation musicale, ce qui ne fut toutefois pas le cas de Jacques VI. Le mariage de Jacques $\mathrm{V}$ en 1537 avec Madeleine de France, fille de François I ${ }^{\text {er }}$, puis, à la mort prématurée de cette dernière, à Marie de Guise, avait renforcé les liens culturels et politiques entre les deux pays. Ronsard, page de douze ans attaché à la suite de Madeleine de France, passa trente-six mois dans les îles britanniques, dont trente en Écosse. De ce séjour nous ne savons malheureusement rien mais la présence du futur poète fait figure de symbole, car Ronsard et la Pléiade devaient laisser leur empreinte sur la production poétique à la Cour de Jacques VI.

Tout en restant féru de poésie italienne et française dans le texte, Jacques VI appréciait aussi « Scottis Poesie », en dialecte scots littéraire, comprenant que par ce moyen pouvait s'affirmer une présence écossaise

9. 9 septembre 1513 .

10. De 1526 à 1528, Jacques V resta quasiment prisonnier d'Archibald Douglas, sixième comte de Douglas, qui plaça ses créatures à tous les postes-clés du pouvoir et de l'Église. En août 1582 Les comtes protestants de Gowrie et Angus attirèrent Jacques VI au château de Ruthven et l'y retinrent captif pendant un an pour le contraindre à chasser d'Aubigny.

11. 1520-1522 : Université de Paris. 1526-1537; reçu Licencié au Collège des Écossais à Paris, puis nommé professeur au Collège Sainte-Barbe; devient précepteur à Paris de Gilbert Kennedy, comte de Cassilis, troisième du nom. 1539-1542 : professeur de latin au Collège de Guyenne à Bordeaux (il eut pour élève un certain Michel de Montaigne); 1544-1547 : nommé professeur au Collège du cardinal Lemoine à Paris ; 1553-1560 [1561?] : nommé professeur au Collège de Boncourt à Paris.

12. Giulio Cesare della Scala (1484-1558), Joseph Justus Scaliger (1540-1609), Marc Antoni Muret (1526-1585), Adrien Turnèbe ou Tournebeuf (1512-1565). Poète lui-même (en langue latine), George Buchanan fut témoin de la querelle littéraire opposant Ronsard et la Pléiade à Mellin de Saint-Gelais. Cependant il serait hasardeux de voir en Buchanan l'introducteur en Écosse des principes poétiques énoncés par la Pléiade. 
spécifique au sein de l'Europe, au moins par des traductions. Le monarque souhaitait maintenir une pratique autochtone tout en ouvrant sa Cour à la vie culturelle européenne.

Esmé Stuart, sire d'Aubigny, exerça un grand ascendant sur son cousin Jacques VI lors de son séjour à la Cour d'Écosse de 1579 à 1582. L'accompagnait une suite brillante composée de gentilshommes accomplis, grands cavaliers, exercés au métier des armes et courtisans modèles. Ce personnage ne pouvait que séduire un jeune homme solitaire élevé dans l'austérité puritaine par un sévère précepteur qui ne ménageait pas les châtiments corporels. C'est pendant la période d'oisiveté forcée au château de Ruthven que le jeune Jacques VI conçoit le style poétique «staitly style » devant caractériser la production et le jeu poétique de son cercle littéraire. Ainsi le souverain-poète se lance-t-il dans la théorie littéraire, comme ses homologues français de la Pléiade quelques décennies plus tôt ${ }^{13}$. En 1583, après que Jacques VI eut recouvré la liberté, Robert Hudson, poète du cercle littéraire royal, reçoit une lettre anonyme, en vers. Celle-ci décrète l'instauration du régime des lettres à la Cour, autour de la métamorphose de l'homme d'arme en homme de lettre troquant sa dague contre une corne à encre et sa cotte de maille, contre du papier. Plutôt que des faits d'arme, écrire des vers va devenir un moyen d'avancement à la Cour : c'est investir la poésie d'une fonction sociale et économique, en sus de sa fonction ludique, spirituelle et symbolique. Notons à cet égard que le jeune Ronsard rêvait à ses débuts de gloire militaire et que son homologue écossais Alexandre Montgomerie s'était acquis une certaine réputation dans la carrière militaire. Les vertus littéraires prétendent dorénavant concurrencer les prouesses guerrières. On célèbrera l'amour courtois, et les vertus chrétiennes sur fond de puritanisme, deux pôles littéraires reflétant les luttes idéologiques et religieuses en Écosse.

\section{Le « Castalian Band » ou « Brothers of the Muses », 1563-1586}

Précisons tout d'abord que «band » en dialecte scots, dénote une association de conjurés ayant fait serment de s'engager dans une action violente. Mais ici le terme se trouve détourné de son sens originel pour désigner un cercle autour du roi s'adonnant à la littérature et à la musique au service d'une cause commune, celle énoncée dans la lettre qu'avait reçue le poète Robert Hudson. La notion de conjuration qu'évoque le vocable «band » reflète peut-être le projet littéraire qu'élabora le roi dans sa solitude lors de son emprisonnement au château de Ruthven et, par la suite, son inten-

13. Défense et Illustration de la langue française (1549), Joaquim Du Bellay. 
tion de contrer des mœurs encore rudes par une entreprise « civilisatrice » fondée sur les arts, entreprise « ourdie » au milieu d'intrigues et de manœuvres politiques incessantes.

$\mathrm{Si}$ « conjuration » il y a, elle n'a plus rien à voir par son pacifisme et ses visées à celle qui aboutit à l'assassinat du second époux de Mary Queen of Scots, Henry Stuart dit Lord Darnley ${ }^{14} \ldots$

Le «Castalian Band » représente un groupe formé autour du souverain, comprenant membres de la famille royale et serviteurs à la Cour ${ }^{15}$. Avec pour arrière-plan des intrigues ourdies par des partis de nobles afin de faire main basse sur le pouvoir, le « Castalian Band » représente une fraternité pacifique, unie par une cause spirituelle. Le délassement joua également sans doute un rôle dans pareille entreprise : l'art fournissait un dérivatif bienvenu aux tracas politiques. Après sa fuite du château de Ruthven, Jacques VI souhaite l'unité de ses nobles, la concorde; il désire se faire respecter pour son impartialité, au milieu d'une Cour où la plupart sont catholiques, certains partisans de la reine Mary emprisonnée à Londres, d'autres encore, en contact avec les Guise en France. À l'occasion de ses vingt-et-un ans, le roi tente de pacifier ses nobles ennemis par un rituel symbolique : il organise une «love-feast » où sont convoqués au même festin des ennemis jurés, priés de se rendre en procession à l'église par deux, main dans la main. Dans cette perspective, le « Castalian Band » représente une tentative d'union politique par un roi œuvrant pour la paix en son royaume. La haute culture devait être l'instrument de cette politique.

L'activité littéraire du "Castalian Band » est à l'origine liée au " puy ${ }^{16}$ »" ou jeu d'échanges écrits qui devint en Écosse « lusus regius » (His Majesty's recreation at vacant hours). Les joutes oratoires et poétiques (incluant l'invective, comme nous l'avons vu) faisaient partie du fonds culturel écossais. La création du "Castalian Band » relève donc d'un phénomène plus largement européen, relevant de la politique tout autant que de la culture.

On trouve parmi ce cercle les poètes écossais (makars) Alexander Montgomerie, William Fowler, William Alexander, John Stewart, Patrick Hume of Polwarth, et Alexander Hume, sans compter les poètes venus d'autres parties des îles britanniques, comme Thomas et Robert Hudson, poètes et musiciens issus du nord de l'Angleterre.

14. 9 février 1567.

15. Rappelons qu'à l'époque, les artistes, poètes ou musiciens, sont considérés comme des serviteurs entièrement tributaires du parrainage des puissants.

16. Les «puys » étaient des sociétés de notables pieux créées au XIII siècle au nord de la France et en Flandre, consacrées à la Vierge. Elles se rassemblaient autour de valeurs spirituelles et culturelles, le caractère profane finissant par s'imposer au fil des siècles. Certaines sociétés devinrent des lieux de joutes poétiques ponctuées par l'élection d'un poète-lauréat. 
Le «Castalian Band » s'organise autour d'une allégorie bien dans l'esprit de la Renaissance : on assimile la personne royale à Apollon, dieu du soleil, de la poésie, des muses, versificateur lui-même et inspirateur des poètes. C'est d'ailleurs en ces termes que Robert Ayton, le dernier « castalien », célèbre Jacques VI, le « roi-pacificateur ». Le " Castalian Band » s'assigne comme fin de créer une nouvelle poésie en dialecte scots de Cour, afin de servir la gloire du roi et le prestige de la nation.

Dans une certaine mesure, on peut trouver des points communs avec le mouvement poétique associé à la Pléiade : ce mouvement voit un groupe de jeunes amis poètes réunis pendant une dizaine d'années autour de la création littéraire française. Ce sont des humanistes, tels que Ronsard, Du Bellay, Pontus de Tyard. Ils se proposent d'enrichir la langue par l'importation de mots grecs et latins; de poser des règles précises de versification; de faire retour aux formes antiques (satire, élégie, épître, comédie, tragédie, épopée); de ne conserver que le sonnet comme forme poétique. En Écosse, le choix de la langue vulgaire («Middle scots») et l'intérêt pour ce véhicule linguistique appartient bien à l'esprit de la Renaissance (Jacques VI, capable de versifier en latin, ne dédaigne pas sa langue maternelle).

Comme chez les poètes de la Pléiade, les compagnons de poésie de Jacques VI usent exclusivement du sonnet et le roi lui-même écrit un traité de poésie (Some Reulis and Cautelis to Be Observit and Eschewit in Scottish Poesie, inclus dans The Essayes of a Prentise, Édimbourg, 1585); le sonnet devient la forme poétique obligée dont on explore toutes les variétés possibles comme l'atteste la production du « Castalian Band », comprenant traductions et créations propres.

Tous les genres poétiques se trouvent représentés, en fonction des neuf muses, parfois par le biais de traductions; chaque membre du groupe se voit attribuer une muse : ainsi le musicien écossais James Lauder et ses danses de Cour sera-t-il naturellement placé sous l'égide de Terpsichore.

Parmi les traductions de l'italien et du français en dialecte scots littéraire on peut citer, pour leur impact sur la vie culturelle écossaise aristocratique :

- Fudith de Du Barthas ${ }^{17}$, traduit par Thomas Hudson, projet lancé au cours d'une conversation de table portant sur le passage de l'hexamètre au pentamètre ${ }^{18}$. Du Bartas fut universellement connu en Europe où il eut un grand rayonnement pendant un demi-siècle (il

17. Poète calviniste (1544-1590).

18. Dans son fournal de voyage en Italie (1580-1581), Montaigne, lors d'un séjour à Rome au mois de mars, mentionne une discussion à table entre lui et ses hôtes (l'ambassadeur français et des « savants » dont l'humaniste et philosophe Marc-Antoine Muret). Contre eux, il défend la traduction française 
y eut des centaine d'éditions, et des dizaines de traductions, après sa mort).

- Triomphes de Pétrarque, traduit par William Fowler.

- Ane Abbregement of Roland Furious translated out of Ariost par John Stewart of Baldyneis pour faire connaître l'Arioste en Écosse ainsi que la poésie épique, par le biais de la traduction française de Desportes intitulée Imitations de l'Arioste.

- Uranie de Du Bartas publiée en 1584, et, du même auteur, une soixantaine de vers de la Sepmaine (poème encyclopédique narrant la Création, 1578), ainsi qu'une cinquantaine tirée de Eden, traduits par Jacques VI lui-même.

Et parmi les compositions originales :

- Lepanto (composé en latin par le roi lui-même) représente la poésie historique, célébrant la bataille navale de Lépante en 1571 au large de la Grèce, qui mit fin à l'expansionnisme ottoman en Méditerranée.

Si la poésie à la Cour de Jacques VI, est au départ, " récréation du roi par la poésie », elle devient désormais un objet destiné à l'imprimerie, promis à circuler dans l'Europe littéraire. Une première impression voit le jour en 1584 à Édimbourg, chez Thomas Vautroullier (ou Vautrollier). Il s'agit de Essayes of a Prentice in the Divine Arte of Poesie, de Jacques VI, contenant la traduction en dialecte scots par Thomas Hudson de la Fudith composée par Du Bartas, avec pour préface un sonnet de Jacques VI et un de Fowler. On trouve également des sonnets à la gloire du roi, par Thomas Hudson (dont un mis en musique), William Fowler et Alexander Montgomerie. Certains sonnets ont pour thème les dieux, d'autres, les saisons, outre une lamentation royale sur la mort du favori, Phenix. En 1591 est publié à Edimbourg His Maiesties Poeticall Exercises at Vacant Houres, manifeste poétique de la main du roi, rappelant la notion de lusus regius. Ce traité évoque le poète parfait, et une version du texte sous forme de psaume. Il s'agit du premier manifeste pour une nouvelle poésie écossaise de la Renaissance. Jacques VI connaissait les théories et œuvres de la Pléiade (Abrégé théorique de Ronsard, poèmes de Ronsard, Du Bellay) mais il connaissait aussi d'autres points de vue sur l'art poétique, comme celui de Du Bartas, le poète français le plus connu en Europe. Les littérateur castaldiens (Montgomerie, Lauder, Thomas Hudson) avaient accès aux sonnets français de la Pléiade. Le roi Jacques VI, féru de poésie française, ne pouvait que leur prêter une oreille bienveillante. Le monarque

de Plutarque (probablement celle de Jacques Amyot). Ceci donne une idée du niveau culturel atteint par l'élite intellectuelle à l'époque. 
souhaitait que la nouvelle poésie se crée en lien avec la nouvelle poésie anglaise et continentale, tout en s'inspirant de la tradition nationale (poésie allitérative médiévale ou " tumbling verse ») considérée comme aussi importante que les nouvelles formes. L'hybridité des productions culturelles écossaises se signale encore ici : l'esprit de l'amour courtois irrigue toujours les poèmes mais se trouve confronté à la montée d'un certain puritanisme; l'ouverture culturelle sur l'Europe va de pair avec la conservation de pratiques poétiques autochtones.

Ce dernier point, l'entreprise de George Bannatyne et Sir Thomas Wode of Saint Andrews l'atteste amplement : Bannatyne compila une anthologie manuscrite de poésie écossaise antérieure à Jacques VI et contemporaine de son règne, The Ballat Manuscript written in tyme of Pest, 1568; Sir Thomas Wode est l'auteur d'une anthologie de compositions musicales sacrées et profanes à plusieurs parties, originaires d'Écosse, d'Angleterre et du continent, Saint Andrews Psalter (1562-1590), Wode Part-books.

L'Écosse prend part à la circulation des hommes et des idées autour de la chose littéraire : Du Bartas partit en ambassade à la Cour de Jacques VI avec le poète Jean de Sponde ${ }^{19}$ en 1584. Jacques VI entretint par ailleurs d'excellentes relations avec d'autres poètes protestants français (il accueillit dans son entourage Jean de la Gessée ${ }^{20}$ ) : en retour, Du Bartas lui dédia le poème La vocation et transposa sous le titre La Lépanthe le poème latin du roi Lepanto. Jean de Schelandre ${ }^{21}$ dédia à Jacques ${ }^{\text {er }}$ d'Angleterre en 1611 La Stuartide en l'honneur de la très-illustre maison des Stuarts, dédiée au Sérénissime roy de la grande Bretaigne.

\section{Influence de Ronsard et de la Pléiade en Écosse}

Le poète de Cour Alexandre Montgomerie mêle l'ancienne tradition poétique écossaise (allitérative, rappelons-le) à la nouvelle poésie française de la Pléiade, et en particulier à celle de Ronsard. Il eut une influence décisive sur la culture de Cour. C'était un jeune homme aimable, plein d'esprit, convivial, capable d'improviser des rimes en des joutes impromptues. Il encouragea l'air de Cour ${ }^{22}$ dans la mesure où il avait le souci d'écrire des

19. 1557-1595. Traducteur d'Homère en latin, auteur de poésies d'abord érotiques, puis d'inspiration chrétienne.

20. 1550-1600. Poète, historien et généalogiste français.

21. 1584 [1585?]-1635. Tragédien, poète élève de Ronsard et de Du Bartas.

22. Air de Cour : poème mis en musique, soit pour voix et luth, soit pour plusieurs voix auxquelles on pouvait substituer ou mélanger des instruments. L'air de Cour anglais (« ayre ») est accompagné au luth (ou à l'orpharion, cousin anglais du luth mais cordé de métal) renforcé par une viole de gambe basse. 
vers « amoene to music ». Les poètes de la Pléiade proposaient souvent dans leurs publications des « airs ${ }^{23} »$ pour mettre leurs oeuvres en musique, un air pouvant convenir à plusieurs sonnets («vers mesurés sur la lyre »), à condition qu'ils aient la même structure rythmique. L'adéquation de la musique à la poésie constitue une des aires de recherche importantes à la Renaissance. Jean Antoine de Baïf, membre de la Pléiade et fondateur en 1570 de l'Académie de musique et de poésie, se propose de soumettre vers et musique à des règles communes. Ainsi se trouve fondé le principe de «musique mesurée à l'antique ", dans laquelle une syllabe longue du texte correspondra à une valeur musicale longue, et inversement, de manière à reproduire la métrique grecque ${ }^{24}$. La musique doit épouser la fluidité rythmique propre au texte, selon les principes de la rhétorique ${ }^{25}$.

Proche du jeune Jacques VI, il renforça l'influence française à la Cour écossaise en tant que poète principal, par des cartels ronsardiens ${ }^{26}$, des élégies imitées de Marot, des chansons et sonnets s'inspirant de la Pléiade. Dès 1582, Montgomerie introduisit le sonnet ronsardien dans la Scottis Poesie après qu'en France on eut publié en 1552 Les amours de Pierre de Ronsard, sonnets mis en musique. C'est cet ouvrage qui inspira les soixantedix sonnets de Montgomerie, séduit par l'alliance intime du vers et de la musique réalisée dans Les amours. Les sonnets adaptés ou traduits de Ronsard étaient à l'origine destinés à être chantés, comme les sonnets de Les amours « mis à quatre parties ${ }^{27}$ » par Lejeune, Jeannequin, Muret, Goudimel, Gerton.

\section{The Gude and Godlie Ballatis or "Compendius Buik" 1578-1600}

Ce recueil, d'abord manuscrit, puis publié à Édimbourg en 1600, contient des œuvres couvrant à dix années près la période du règne en Écosse de Jacques VI. Il illustre la Réforme dans le royaume et contient l'équivalent des «psautiers » huguenots continentaux. Par exemple, on y trouve

23. Désigne à l'époque des arrangements polyphoniques à quatre ou cinq parties.

24. Participèrent à ce projet les musiciens Claude Le Jeune (1530-1600), Eustache du Caurroy (1549-1609), Jacques Mauduit (1557-1627), Guillaume Costeley (1531-1606), Nicolas de la Grotte (1530-1600).

25. C'est la règle pour l'air de Cour français dans la première moitié du XVII ${ }^{\mathrm{e}}$ siècle. L'application de la métrique rhétorique à l'interprétation actuelle de la musique ancienne est depuis longtemps un principe directeur.

26. Comme son nom le suggère, le « cartel » poétique est une charge en vers dirigée contre une personne ou idée. En effet, dans un sens maintenant obsolète, le terme désignait concrètement un petit carton sur lequel on rédigeait une provocation en duel, d'où par métonymie, la provocation elle-même. Le cartel appelle une réponse, il va sans dire.

27. Expression consacrée à l'époque pour désigner une composition polyphonique à quatre voix. 
une version écossaise des noëls allemands In dulci jubilo ou encore Vom himel hoch da kom ich her (composé par Luther) sur un air de berceuse écossaise, Balulalow ${ }^{28}$. Y figurent également des cycles de chants de Noël et de Pâques, ainsi que des « airs de Cour » dans lesquels l'amour terrestre cède la place à l'amour divin, comme par exemple for Life of One adapté de Dont vient cela de Sermisy et Marot, illustrant la tension spécifiquement écossaise entre amour terrestre et amour spirituel. Dans le recueil écossais l'esprit de l'amour courtois s'estompe au bénéfice de pièces à caractère uniquement spirituel. À preuve, la moitié des « spirituall songis » consiste en traductions et adaptations de pièces allemandes ou scandinaves, provenant donc de pays touchés par la Réforme. Le but est de chanter les évangiles et la doctrine réformée sur une musique souvent empruntée à des chansons profanes, dans la tradition du contrafactum.

\section{L'influence poétique française en Écosse par l'exemple : commentaire de l'adaptation de Dont vient cela (poème de Marot sur une musique de Sermisy) par Alexander Scott}

Alexander Scott offre un intéressant exemple d'adaptation linguistique et musicale à partir du poème Dont vient cela. L'avatar en scots littéraire colle parfaitement à l'air d'origine. Le poète écossais ne livre pas une traduction littérale cependant et fait subir à l'original de Clément Marot un traitement particulier, comme on le verra ci-dessous :

\section{Original}

Dont vient cela, belle, je vous supply,

Que plus à moy ne vous recommandez?

Tousjours seray de tristesse remply,

Jusques a tant qu'au vray me le mandez.

Je croy que plus d'amy ne demandez.

Ou maulvais bruyt de moy on vous revelle

Ou vostre cœur a fait amour nouvelle.

S vous laissez d'amour le train joly,

Vostre beauté prisonniere rendez

Si pour autruy m'avez mis en oubly,

Dieu vous y doint le bien que pretendez;

Mais si de mal en rien m'apprehendez,

28. Il n'y a rien d'étonnant à ce qu'un air de berceuse se voit affecté de paroles pieuses : il s'agit d'une pratique séculaire, celle de la «parodie » ou contrafactum », en Écosse comme sur le continent. 
Je veule qu'autant que vous me semblez belle, D'autant ou plus vous me soyez rebelle.

* Poème de Clément Marot (1486/1497-1544)

* Musique de Claudin de Sermisy (v. 1490-1562)

NB. Marot reprendra la musique pour son psaume X, Dont vient cela, Seigneur, je vous supply (« parodie » de l'original, auquel on substitue des paroles pieuses).

\section{Adaptation}

For lov of one I mak my moan, right secretly, To Venus queen, that ladie sheen, for remedie, For remedie,

Or I be dead send some remead, of your mercie, For him that wroght us all of nought my fair ladie, For him, that wroght us all of nought my fair ladie

To cause that she grant grace to me ere I begon For to redresse my hevinesse and ease my mone, And ease my mone,

Or I be dead send some remead, of your mercie, For him that wroght us all of nought my fair ladie, For him, that wroght us all of nought my fair ladie.

Comfort is non but mourn alon for my mistres But if that she wold heav pitie on my distresse It is my joy to thol anoy for my ladie Both woe and pane in sorowe slaine qhill that I die, Or I be dead send some remead, of your mercie, For him that wroght us all of nought my fair ladie, For him, that wroght us all of nought my fair ladie.

Qher ever I gang that is my sang qhill I may lest Unto that queen qho ay has been my herts sole rest My fair mistresse of your goodnesse some comforting Without your grace adew solace welcom mourning, Or I be dead send some remead, of your mercie, For him that woght us all of nought my fair ladie, For him, that wroght us all of nought my fair ladie.

* Poème de Alexander Scott (1520[?]-1582/1583)

Wode Part-Books.

On remarquera de prime abord ceci : l'adaptation paraît sensiblement plus longue que l'original (vingt-six lignes contre quatorze). Cela tient au 
fait que Alexander Scott introduit un refrain («Or I be dead send some remead, of your mercie, / For him that wroght us all of nought my fair ladie ») dont la seconde ligne se trouve répétée, à la fin de chaque strophe. Si l'on élimine ce refrain, on retrouve les quatorze vers de l'original.

D'un point de vue thématique, nous avons bien une adaptation et non une traduction littérale :

- L'original voit s'interroger l'amant sur l'indifférence que semble lui témoigner nouvellement sa belle. A-t-elle renoncé à l'amour? («Je croy que plus d'amy ne demandez »); lui a-t-on dit du mal de l'amant? («Ou maulvais bruyt de moy on vous revelle ») A-t-elle jeté son dévolu sur un autre? («Ou vostre cœur a fait amour nouvelle ») La deuxième strophe contient les réponses que donne l'amant à ses trois questions, mais dans un ordre différent. On notera que l'amant, dans l'hypothèse où sa belle n'aurait pas eu de fâcheux échos de lui, se déclare prêt à relever le défi de la séduction («Mais si de mal en rien m’apprehendez / Je veule qu'autant que vous me semblez belle / D'autant ou plus vous me soyez rebelle»). La tonalité de l'ensemble n'est pas du tout celle de maintes chansons d'amour de l'époque, dans lesquelles l'amant se répand en lamentations : ici on a plutôt un discours amoureux fondé sur le raisonnement et l'argumentation rationnelle, dans l'esprit de l'amour courtois (« Si vous laissez d'amour le train joly / Vostre beauté prisonniere rendez » — « Si pour autruy m'avez mis en oubly / Dieu vous y doint (= donne) le bien que pretendez »).

- Dans l'adaptation que réalise Alexander Scott, l'« air de Cour » tourne autour du thème unique de la supplique adressée à la déesse de l'amour Vénus («... I mak my moan, ... To Venus queen ... ») : l'amant malheureux («For lov of one ... ») cherche un réconfort (« to redresse my hevinesse and ease my mone ... »). Il supplie Vénus de toucher le coeur de sa bien-aimée («But if that she wold heav pitie on my distresse ») car le désespoir menace sa vie (« ... ease my mone / Or I be dead $\left.{ }^{29} »\right)$. Il y a donc changement thématique, l'air se concentrant désormais sur l'expression de la seule souffrance amoureuse exempte de tout mode de « raisonnement » à caractère rhétorique. L'esprit de l'amour courtois se trouve manifestement contrebalancé

29. Aujourd'hui la figure de l'amant désespéré aux portes de la mort nous semble extrêmement convenue, , voire vidée de tout sens. Convention littéraire mise à part, il convient de se rappeler qu'aux $\mathrm{XVI}^{\mathrm{e}}$ et XVII ${ }^{\mathrm{e}}$ siècles, comme l'attestent, entre autres, les mémorialistes, les passions, peuvent avoir sur l'organisme humain des effets parfois violents à l'époque : on peut se pâmer (littéralement) en entendant des frottements harmoniques dans un passage musical, mourir (littéralement) de chagrin, ou à tout le moins tomber physiquement malade (nous dirions aujourd'hui « somatiser »). 
par les références divines formant refrain. Si Marot mentionne Dieu, c'est dans le cadre d'une expression figée («Dieu vous donne... ») dont les connotations spirituelles sont faibles. Par contre, Alexander Scott place la prière à Vénus sous l'égide de Dieu tout puissant, au nom de qui («For him that wroght us all of nought») l'amant sollicite la pitié. On a donc ici l'expression de sentiments chrétiens (la compassion, la miséricorde) coulés dans le moule du paganisme (la prière à Vénus) bien dans l'esprit de la Renaissance, et de la Renaissance écossaise tout particulièrement. Cependant la réitération induite par le refrain rééquilibre l'ensemble dans un sens sensiblement plus spirituel que l'original : chez Scott, qui assura la continuité entre le règne de Jacques V, celui de Mary Queen of Scots et celui de Jacques VI, l'esprit courtois cède en partie à l'expression de la souffrance et de la miséricorde divine. Peut-être faut-il voir dans cet avatar écossais de Dont vient cela un symptôme de la tension intellectuelle caractéristique d'un pays en proie aux affres de la Réforme et de la contre-Réforme.

D'un point de vue musical, on n'observe aucun changement par rapport à Sermisy : Scott, auteur d'« airs de Cour », a manifestement privilégié le principe de poesie amoene to music au prix d'une grande liberté d'adaptation, elle-même vraisemblablement tributaire du climat intellectuel régnant à la Cour de Jacques VI.

\section{Conclusion}

On considère Sir Robert Ayton comme le dernier grand poète et auteur d'« airs de Cour » héritier du «Castalian Band ». Longtemps fort apprécié dans l'entourage de Jacques ${ }^{\text {er }}$ et de Charles I $^{\text {er }}$, il fut ami de Ben Johnson, du musicien-poète Thomas Campion et secrétaire des reines Anne et Henrietta Maria. Avec l'avènement de Charles ${ }^{\text {er }}$ le dialecte scots passa de plus en plus pour un langage rustre : on anglicisa donc les productions composées dans cet idiome, productions qui devinrent le fleuron poétique des « Cavaliers ». Ceux-ci se firent les héritiers d'une poésie courtoise, contre l'austérité puritaine.

La Cour des souverains écossais atteste une vie culturelle florissante, hélas trop intermittente. Les témoignages de l'ambassadeur espagnol Don Pedro de Ayala ${ }^{30}$, du poète William Dunbar, ainsi que les comptes de la trésorerie sous Jacques IV constituent autant de preuves indiquant la

30. Ambassadeur permanent de Ferdinand II d'Aragon et d'Isabelle I ${ }^{\text {re }}$ de Castille à la Cour de Jacques IV (1496-1497). 
présence d'un nombre non négligeable de musiciens, dont ceux de la Chapelle royale refondée en 1501. C'est l'époque où éclot la musique sacrée composée par Robert Carver, chanoine de Scone; mais il ne reste pas trace de la musique profane créée à cette époque-là. La fin tragique du roi sur le champ de bataille suspendit la vie culturelle de la Cour jusqu'à la majorité de Jacques V en 1528. Sous le règne, de ce dernier, danse et chansons, mimes, épîtres galantes, joutes d'esprit, chansons d'amour et satires obscènes reprirent leurs droits. Les mariages politiques du souverain avec des princesses françaises à partir de 1536 renforcèrent les liens culturels avec la France, prépondérants, mais n'empêchèrent pas cependant la présence de musiciens anglais, et surtout italiens. Ce fut de nouveau l'éclipse, de 1542 (mort du roi) jusqu'à 1579, date à laquelle Jacques VI atteint sa majorité et redonna à sa Cour une vie culturelle digne de ce nom.

Avec le départ de celle-ci pour Londres, la tradition de l' " air de Cour » et de l'« air à plusieurs voix » se prolongea en Écosse jusque vers la fin du $\mathrm{XVII}^{\mathrm{e}}$ siècle, dans les châteaux et les lieux de savoir mais d'une manière conservatrice, la Cour n'étant plus là pour inspirer des changements en matière d'esthétique. On observe un phénomène comparable quant aux six manuscrits de musique pour luth, dans lesquels se trouvent mis en tablature ${ }^{31}$ de plus en plus d'airs populaires du cru, les échanges musicaux avec le continent s'étant taris.

En 1707, l'Écosse perdit son parlement et connut un sursaut de nationalisme culturel, voyant dans la vieille Scottis Poesie l'image d'une grandeur passée. Allan Ramsey collecta dans son recueil Evergreen de la poésie du $\mathrm{XVI}^{\mathrm{e}}$ siècle véhiculée par le dialecte scots littéraire. Comme George Bannatyne et Thomas Wode avant lui, il ne fit que conserver une production culturelle désormais figée. Le poème allégorique d'Alexander Montgomerie, the Cherrie and the Slae, déjà passablement anglicisé depuis sa première parution au XVI ${ }^{\mathrm{e}}$ siècle retrouva sous la plume de Ramsey sa forme archaïsante — elle en fit d'ailleurs le succès — et attira plus tard l'attention d'un certain Robert Burns, qui s'inspira de ses rythmes. Cependant il n'en alla pas de même des pièces musicales polyphoniques, mélangées à tant d'œuvres anglaises que peut-être on ne les estima pas assez dignes d'une renaissance culturelle « nationale ». Seules trouvèrent grâce les chansons monophoniques présentées comme « autochtones ».

Un air connu parmi les «Cavaliers », Old Lang Syne, avec des paroles attribuées à Sir Robert Ayton, a fait le tour du monde pour ponctuer le moment où l'on doit se quitter (depairt). C'est le dernier écho du «Cas-

31. La musique pour luth passe par une tablature de six lignes agrémentées de lettres ou de chiffres indiquant les cases où mettre les doigts. 
talian Band », mais sous l'aspect que lui donna Robert Burns. Celui-ci reprit à son compte la mélodie ancienne, mise toutefois au goût du jour, sur laquelle il composa la chanson Should auld acquaintance be forgot, inspirée d'une version par James Watson (1711). Ultime avatar, c'est ce texte qui se chante aujourd'hui... sur un air écossais différent de l'original!

\section{Discographie}

— Les cris de Paris, ensemble Clément Janequin («Dont vient cela »), direction Dominique Visse (contre-ténor), Michel Laplénie (ténor), Philippe Cantor (baryton), Antoine Sicot (basse), Harmonia Mundi, coll. «Musique d'abord», HMA 1951072, 1982-2005.

- Notes of Noy / Notes of Foy, The Rowallan Consort (" For Love of One »), direction Robert Phillips (luth), William Taylor (harpe écossaise ou clarsach), Mhairi Lawson (soprano), Paul Rendall (Ténor), Temple Records, COMD 2058, 1994.

\section{Bibliographie}

Cordner Michael (éd.), Court Masques, coll. « Oxford World's Classics/ Oxford English Drama », Londres, Oxford University Press, 1998.

ElLiott Kenneth (éd.), Mennie Shire Helena (pour les textes des « airs de Cour »), Music of Scotland 1500-1700, coll. « Musica Britannica », tome XV, Londres, Stainer \& Bell, 1957, p. 140-188.

Mennie Shire Helena, Song, Dance \& Poetry of the Court of Scotland under King James VI, Cambridge, Cambridge University Press, 1969. 